\title{
EXPONENTIAL MIXING FOR SOME SPDES WITH LÉVY NOISE
}

\author{
ENRICO PRIOLA, LIHU XU, AND JERZY ZABCZYK
}

\begin{abstract}
We show how gradient estimates for transition semigroups can be used to establish exponential mixing for a class of Markov processes in infinite dimensions. We concentrate on semilinear systems driven by cylindrical $\alpha$-stable noises introduced in [17, $\alpha \in(0,2)$. We first prove that if the nonlinearity is bounded, then the system is ergodic and strong mixing. Then we show that the system is exponentially mixing provided that the nonlinearity, or its Lipschitz constant, are sufficiently small.
\end{abstract}

Keywords: stochastic PDEs driven by $\alpha$-stable noises, ergodicity, strong mixing, exponential mixing.

Mathematics Subject Classification (2000): 60H15, 47D07, 60J75, 35R60.

\section{INTRODUCTION}

The paper is concerned with asymptotic properties of the stochastic evolution equation:

$$
d X(t)=[A X(t)+F(X(t))] d t+d Z_{t}, \quad X_{0}=x,
$$

driven by $\alpha$-stable, $\alpha \in(0,2)$, cylindrical process $Z$, introduced in [17]. The paper [17] investigated structural properties of $X$ like strong Feller and irreducibility. Solutions turned out to be stochastically continuous but in general without cádlág modifications, see [4]. The stochastic PDEs driven by Lévy noises have been intensively studied since some time, see e.g. [3], [1], [16], [13], [12], [17] and [20], and the book [15] for additional references. Even for equations like (1.1), with the additive noise, some basic questions are still open.

General results on existence of invariant measures for the linear equation

$$
d X(t)=A X(t) d t+d Z_{t}, \quad X_{0}=x
$$

The first author gratefully acknowledges the support by the M.I.U.R. research project Prin 2008 "Deterministic and stochastic methods in the study of evolution problems". The second author gratefully acknowledges the support from EURANDOM and Hausdorff Research Institute for Mathematics. The third author gratefully acknowledges the support by Polish Ministry of Science and Higher Education grant "Stochastic equations in infinite dimensional spaces" N N201 419039 . 
with $A$ being the infinitesimal generator of a strongly continuous semigroup, were obtained in [6], and more recently in [18] and [10], with different assumptions on $Z$. The nonlinear case was investigated in [19], and by the dissipativity method in [15]; see also the recent paper [20].

In the present paper we are concerned with the exponential convergence to equilibrium, called exponential mixing, see e.g. [15, Proposition 16.5] .We assume that the nonlinearity $F$ is Lipschitz continuous and bounded and find explicit conditions on $F$ under which the exponential mixing takes place. The speed of the convergence to equilibrium can be deduced from the proofs. Our result seems to be new even in the well-studied case when $Z$ is a cylindrical Wiener process, corresponding to $\alpha=2$, requiring only obvious modifications of our proofs.

To prove the exponential mixing we establish first gradient estimates for the transition semigroup of the process $X$ using the so called mild Kolmogorov equation. This version of the Kolmogorov equation was introduced in [5] and elaborated in [7] and [8]. With a similar aim gradient estimates were used in [20], in a different framework, with the assumption $\alpha \in(1,2)$ and without the exponential bounds. The ergodicity in [20] and [21] was obtained by the technique of interacting particle systems called finite speed of propagation of information. For the finite dimensional stochastic system driven by $\alpha$-stable processes, [20] obtained the strong mixing result for small $F$.

Our main results are formulated as Theorems 2.5 and 2.6. The first one establishes ergodicity without additional condition on $F$. Here it was enough to use the method sketched in [9]. Theorem 2.6 is on exponential mixing with additional restrictions on $F$. If the nonlinearity is only Lipschitz and bounded (and not small), it seems hard to obtain exponential mixing by the gradient estimates. We are now working on this general case trying a different method.

The organization of the paper is as follows. Section 2 introduces the notations and the main theorems. Section 3 proves the ergodicity and strong mixing for bounded nonlinearity. The next section is devoted to exponential gradient estimates. In the final section, we prove exponential mixing for small nonlinearities.

\section{Notations And MAin Results}

We shall study our problem in a separable Hilbert space $H$ with an orthonormal basis $\left\{e_{k}\right\}_{k \geq 1}$. Denote the inner product and norm of $H$ by $\langle\cdot, \cdot\rangle_{H}$ and $|\cdot|_{H}$ respectively. Let $C_{b}(H, H)$ be the Banach space of all bounded continuous functions $f: H \rightarrow H$ with the supremum norm

$$
\|f\|_{0}:=\sup _{x \in H}|f(x)|_{H}
$$

Similarly, $C_{b}(H, \mathbb{R})$ denotes the Banach space of all bounded continuous functions $f: H \rightarrow \mathbb{R}$ with the supremum norm $\|f\|_{0}:=\sup _{x \in H}|f(x)|$. 
Given $f \in C_{b}(H, \mathbb{R})$ and $x \in H$, for any $h \in H$, we write

$$
D_{h} f(x):=\lim _{\varepsilon \rightarrow 0} \frac{f(x+\varepsilon h)-f(x)}{\varepsilon}
$$

provided the the above limit exists. If $h \mapsto D_{h} f(x)$ is linear and $\left|D_{h} f(x)\right| \leq C|h|_{H}$, $h \in H$, then there exists a unique element in $H$, denoted by $D f(x)$, such that $D_{h} f(x)=\langle D f(x), h\rangle_{H}$. Clearly, $D f$ is a function from $H$ to $H$. Denote by $C_{b}^{1}(H, \mathbb{R})$ the set of all bounded differentiable functions $f: H \rightarrow \mathbb{R}$ with norm

$$
\|f\|_{C_{b}^{1}}:=\sup _{x \in H}|f(x)|+\sup _{x \in H}|D f(x)|_{H} .
$$

Let $B_{b}(H, \mathbb{R})$ be the set of all bounded (Borel) measurable functions from $H$ into $\mathbb{R}$.

Further, we denote by $\mathcal{D}$ the set of all bounded continuous cylindrical functions $f: H \rightarrow \mathbb{R}$, i.e., $f$ is some bounded continuous function depending on finite number of coordinates of $H$. For any $f \in \mathcal{D}$, denote by $\Lambda(f)$ the localization set of $f$, i.e. $\Lambda(f)$ is the smallest set $\Lambda \subset \mathbb{N}$ such that $f \in C_{b}\left(\mathbb{R}^{\Lambda}, \mathbb{R}\right)$. Finally, define $\mathcal{D}^{1}:=\mathcal{D} \cap C_{b}^{1}(H, \mathbb{R})$.

Let $z(t)$ be the normalized one dimensional symmetric $\alpha$-stable process, $0<$ $\alpha<2$, with the following characteristic function

$$
\mathbb{E}\left[e^{i \lambda z(t)}\right]=e^{-t|\lambda|^{\alpha}}, \lambda \in \mathbb{R} .
$$

The density of $z(1)$ will be denoted by $p_{\alpha}$. The infinitesimal generator $\partial_{x}^{\alpha}$ of the process is of the form

$$
\partial_{x}^{\alpha} f(x)=\frac{1}{C_{\alpha}} \int_{\mathbb{R}} \frac{f(y+x)-f(x)}{|y|^{\alpha+1}} d y
$$

with $C_{\alpha}=-\int_{\mathbb{R}}(\cos y-1) \frac{d y}{|y|^{1+\alpha}}$, see [2].

We will consider equation (1.1) under the following assumptions:

\section{Assumption 2.1.}

- A is a dissipative operator defined by

$$
A=\sum_{k \geq 1}\left(-\gamma_{k}\right) e_{k} \otimes e_{k}
$$

$0<\gamma_{1} \leq \gamma_{2} \leq \cdots \leq \gamma_{k} \leq \cdots$ and $\gamma_{k} \rightarrow \infty$ as $k \rightarrow \infty$

- $F: H \rightarrow H$ is Lipschitz and bounded.

- $Z=\left(Z_{t}\right)$ is a cylindrical $\alpha$-stable process with $Z_{t}=\sum_{k>1} \beta_{k} z_{k}(t) e_{k}$ where $\left\{z_{k}(t)\right\}_{k \geq 1}$ is a sequence of i.i.d. symmetric $\alpha$-stable processes (defined on some fixed stochastic basis $\left(\Omega, \mathcal{F},\left(\mathcal{F}_{t}\right)_{t \geq 0}, \mathbb{P}\right)$ with $0<\alpha<2$ and $\beta_{k}>0$, $k \geq 1$, and

$$
\sum_{k \geq 1} \frac{\beta_{k}^{\alpha}}{\gamma_{k}}<+\infty
$$


- There exists $\sigma \in(0,1)$ such that

$$
\sup _{k \geq 1} \frac{\gamma_{k}^{\frac{1}{\alpha}-\sigma}}{\beta_{k}}<+\infty
$$

Remark 2.2. Note that $A$ generates a strongly continuous semigroup of compact contractions $\left(e^{t A}\right)$ on $H$. Moreover, $e^{t A} e_{k}=e^{-t \gamma_{k}} e_{k}, k \geq 1$.

The first three conditions imply the existence of the unique mild solution $X=$ $(X(t, x))$ to (1.1), see [17]. This is a predictable $H$-valued stochastic process, depending on $x \in H$, such that, for any $t \geq 0, x \in H$, it holds ( $\mathbb{P}$-a.s.):

$$
X(t, x)=e^{t A} x+\int_{0}^{t} e^{(t-s) A} F(X(s, x)) d s+Z_{A}(t), \quad \text { with } Z_{A}(t)=\int_{0}^{t} e^{(t-s) A} d Z_{s} .
$$

Condition (2.6) is used in [17, Section 5] to get the strong Feller property for the Markov semigroup $P_{t}$ associated with the solution $X$ of (1.1):

$$
P_{t} f(x)=\mathbb{E}[f(X(t, x))], \quad f \in B_{b}(H, \mathbb{R}), \quad x \in H, t \geq 0 .
$$

For further use we need an equivalent formulation of (2.6).

Lemma 2.3. For arbitrary $\sigma>0$ the following two conditions are equivalent:

$$
\begin{gathered}
B=\sup _{k \geq 1} \frac{\gamma_{k}^{\frac{1}{\alpha}-\sigma}}{\beta_{k}}<+\infty, \\
k_{t}=\sup _{k \geq 1} \frac{e^{-\gamma_{k} t} \gamma_{k}^{1 / \alpha}}{\beta_{k}} \leq \frac{\hat{c} e^{-\gamma_{1} t / 2}}{t^{\sigma}}, t>0,
\end{gathered}
$$

where $\hat{c}=B 2^{(2 / \alpha)-\sigma} \frac{\sigma^{\sigma}}{e^{\sigma}}$.

Proof. To establish the equivalence one can argue as in the beginning of [17, Section 5]. We only note the following estimate, for any $n \geq 1$,

$$
\frac{e^{-\gamma_{n} t} \gamma_{n}^{1 / \alpha}}{\beta_{n}} \leq 2^{\frac{1}{\alpha}} \exp \left(-\gamma_{1} t / 2\right) \frac{e^{-\frac{\gamma_{n}}{2} t}\left(\gamma_{n} / 2\right)^{1 / \alpha}}{\beta_{n}} .
$$

According to [17], we have the following result for the system (1.1).

Theorem 2.4. Under the Assumption 2.1 there exists a unique mild solution $X(t, x)$ for (1.1). Moreover its associated transition semigroup $P_{t}$ is strong Feller and irreducible.

This paper aims to study the long time behaviour of the system (1.1). The two main theorems are as follows. 
Theorem 2.5. Under Assumption 2.1 there exists a unique invariant measure $\mu$ for the system (1.1). The measure $\mu$ is strong mixing, i.e.

$$
\lim _{t \rightarrow \infty} P_{t} f(x)=\mu(f),
$$

for all $f \in B_{b}(H, \mathbb{R})$ and $x \in H$.

In the formulation of the next theorem $p_{\alpha}$ stands for the density of $z(1)$ and the constant $\hat{c}$ was introduced in Lemma 2.3.

Theorem 2.6. Assume that Assumption 2.1 holds and that one of the following two conditions holds:

(i) $L_{F}<\gamma_{1}$, where $L_{F}$ is the best Lipschitz constant of F;

(ii) $\|F\|_{0}<\frac{C_{0}}{\Gamma(1-\sigma)}\left(\frac{\gamma_{1}}{2}\right)^{1-\sigma}$, where $C_{0}=\hat{c} \int_{\mathbb{R}} \frac{\left(p_{\alpha}^{\prime}(z)\right)^{2}}{p_{\alpha}(z)} d z$.

Then the system (1.1) is exponentially mixing. More precisely there exist constants $C=C\left(|x|_{H}, \alpha,\left(\beta_{n}\right),\left(\gamma_{n}\right), F\right)>0$ and $c=c\left(\alpha,\left(\beta_{n}\right),\left(\gamma_{n}\right), F\right)>0$ such that

$$
\left|P_{t} f(x)-\mu(f)\right| \leq C e^{-c t}\|f\|_{C_{b}^{1}}
$$

for all $f \in C_{b}^{1}(H, \mathbb{R})$ and $x \in H$.

We do not give explicit formulas for the constants $C$ and $c$ but they can be obtained by a careful examination of the proofs.

Let us give some examples which the two main theorems can be applied to.

Example 2.7. Consider the following stochastic semilinear equation on $D=$ $[0, \pi]^{d}$ with $d \geq 1$ with Dirichlet boundary conditions

$$
\left\{\begin{array}{l}
d X(t, \xi)=[\Delta X(t, \xi)+F(X(t, \xi))] d t+d Z_{t}(\xi) \\
X(0, \xi)=x(\xi) \\
X(t, \xi)=0, \quad \xi \in \partial D
\end{array}\right.
$$

where $Z_{t}$ and $F$ are both specified below. It is clear that $\Delta$ with Dirichlet boundary condition has the following eigenfunctions

$$
e_{k}(\xi)=\left(\frac{2}{\pi}\right)^{\frac{d}{2}} \sin \left(k_{1} \xi_{1}\right) \cdots \sin \left(k_{d} \xi_{d}\right), \quad k \in \mathbb{N}^{d}, \xi \in D .
$$

It is easy to see that $\Delta e_{k}=-|k|^{2} e_{k}$, i.e. $\gamma_{k}=|k|^{2}=k_{1}^{2}+\ldots+k_{d}^{2}$, for all $k \in \mathbb{N}^{d}$. We study the dynamics (2.11) in the Hilbert space $H=L^{2}(D)$ with orthonormal basis $\left\{e_{k}\right\}_{k \in \mathbb{N}^{d}} . Z=\left(Z_{t}\right)$ is some cylindrical $\alpha$-stable noises which, under the basis $\left\{e_{k}\right\}_{k}$, is defined by

$$
Z_{t}=\sum_{k \in \mathbb{N}^{d}}|k|^{\beta} z_{k}(t) e_{k}
$$

where $\left\{z_{k}(t)\right\}_{k}$ are i.i.d. symmetric $\alpha$-stable processes with $\alpha \in(0,2)$ and $\beta$ a real number. Note that $\sum_{k \in \mathbb{N}^{d}} \frac{|k|^{\beta \alpha}}{|k|^{2}}<\infty$ if and only if $2>d+\alpha \beta$. 
From Theorems 2.5] and 2.6, we have

- If $F$ is a bounded Lipschitz function and

$$
2>d+\alpha \beta, \frac{1}{\alpha}-\frac{\beta}{2}<1
$$

or equivalently,

$$
\frac{d}{\alpha}<\frac{2}{\alpha}-\beta<2,
$$

then the system (2.11) is strongly mixing.

- If in addition $\|F\|_{0}$ is sufficiently small then the system (2.11) is exponentially mixing.

\section{Proof of Theorem 2.5}

According to Sections 5.2 and 5.3 of [17], the system (1.1) is irreducible and strong Feller. By Doob's theorem (see [8, Theorem 4.2.1]), to prove Theorem 2.5. one only needs to show the existence of invariant measures. To this purpose it is enough to establish the tightness of $\{\mathcal{L}(X(t, x))\}_{t>1}$ for some $x \in H$ (here $\mathcal{L}(X(t, x))$ denotes the law of the random variable $X(t, x)$, see (2.7)). For this we basically follow 9 .

Fix any $x \in H$ and set $X(t, x)=X(t)$. First note that for any fixed $t>0$, $e^{A t}$ is a contraction and a compact operator. Contraction property is clear by the assumption of $A$. The compactness is an easy corollary of the fact that the eigenvalues $-\gamma_{k}$ tend to $-\infty$.

According to [17, Proposition 4.2], for any (small) $\varepsilon>0$, there exists some $M=M_{\epsilon}>0$ such that

$$
\mathbb{P}\left\{\left|Z_{A}(t)\right|_{H} \leq M\right\} \geq 1-\varepsilon
$$

uniformly for all $t \geq 0$. By the assumptions on $F$ and $A$, one clearly has $\left|\int_{0}^{t} e^{A(t-s)} F(X(s)) d s\right|_{H} \leq C$ uniformly for $t \geq 0$ and $\omega \in \Omega$. Hence,

$$
\mathbb{P}\left\{|X(t)|_{H} \leq|x|+C+M\right\} \geq \mathbb{P}\left\{\left|Z_{A}(t)\right|_{H} \leq M\right\} \geq 1-\varepsilon .
$$

Let us rewrite (2.7) as

$$
X(t)=e^{A} X(t-1)+\int_{t-1}^{t} e^{A(t-s)} F(X(s)) d s+\int_{t-1}^{t} e^{A(t-s)} d Z_{s},
$$

thanks to the compactness of $e^{A}$ and (3.1), the family $\mathcal{L}\left(\left\{e^{A} X(t-1)\right\}\right)_{t \geq 1}$ is tight. The integrals $\int_{t-1}^{t} e^{A(t-s)} d Z_{s}$ have the same law as $Z_{A}(1)$ for all $t \geq 1$, and thus their laws are of course tight.

To complete the proof of tightness of $\{\mathcal{L}(X(t))\}_{t \geq 1}$, it is enough to show that the values of the integrals $\int_{t-1}^{t} e^{A(t-s)} F(X(s)) d s$ are contained in a compact set, for any $t \geq 1$. To this purpose note that the operator $\mathcal{R}$ from $L^{2}(0,1 ; H)$ into $H$

$$
\mathcal{R} \phi=\int_{0}^{1} e^{A s} \phi(s) d s, \phi \in L^{2}(0,1 ; H),
$$


is compact (this follows from the compacteness of the operators $e^{A t}, t>0$ ). Using the compactness of $\mathcal{R}$ and the fact that the transformation $F$ is bounded, we get that the integrals are contained in a fixed compact set. The proof is complete.

\section{Exponential gRAdient estimates}

To establish Theorem 2.6 we first derive exponential gradient estimates for the Galerkin approximation of the equation (1.1).

4.1. Galerkin approximation. Let $\left\{e_{k}\right\}_{k \geq 1}$ be the orthonormal basis associated with the operator $A$ and write $\Gamma_{N}:=\{1, \ldots, N\}$

For any $x \in H$ and any integer $N>0$ we have the following approximation of Eq. (1.1):

$$
\left\{\begin{array}{l}
d X_{k}^{N}(t)=\left[-\gamma_{k} X_{k}^{N}(t)+F_{k}^{N}\left(X^{N}(t)\right)\right] d t+\beta_{k} d z_{k}(t), \\
X_{k}^{N}(0)=x_{k},
\end{array}\right.
$$

for all $k \in \Gamma_{N}$, where $x^{N}=\left(x_{k}\right)_{k \in \Gamma_{N}}, x_{k}=\left\langle x, e_{k}\right\rangle_{H}$, and $F_{k}^{N}\left(x^{N}\right)=\left\langle F\left(x^{N}, 0\right), e_{k}\right\rangle_{H}$. Eq. (4.1) can be written in the following vector form

$$
\left\{\begin{array}{l}
d X^{N}(t)=\left[A X^{N}(t)+F^{N}\left(X^{N}(t)\right)\right] d t+d Z_{t}^{N}, \\
X^{N}(0)=x^{N}
\end{array}\right.
$$

where $X^{N}(t)=\left(X_{k}^{N}(t)\right)_{k \in \Gamma_{N}}$ and $Z_{t}^{N}=\left(\beta_{k} z_{k}(t)\right)_{k \in \Gamma_{N}}$. The infinitesimal generator of $(4.2)$ is

$$
\begin{aligned}
\mathcal{L}_{N} & =\sum_{k \in \Gamma_{N}} \beta_{k}^{\alpha} \partial_{k}^{\alpha}+\sum_{k \in \Gamma_{N}}\left[-\gamma_{k} x_{k}+F_{k}^{N}\left(x^{N}\right)\right] \partial_{k} \\
& =\sum_{k \in \Gamma_{N}}\left[\beta_{k}^{\alpha} \partial_{k}^{\alpha}-\gamma_{k} x_{k} \partial_{k}\right]+\sum_{k \in \Gamma_{N}} F_{k}^{N}\left(x^{N}\right) \partial_{k},
\end{aligned}
$$

where $\partial_{k}=\partial_{x_{k}}$ and $\partial_{k}^{\alpha}=\partial_{x_{k}}^{\alpha}$. In the sequel we will identify $x^{N} \in \mathbb{R}^{N}$ with

$$
x^{N}=\sum_{k=1}^{N} x_{k} e_{k} \in H .
$$

Consider the Kolmogorov equation of the Galerkin approximation

$$
\left\{\begin{array}{l}
\partial_{t} u^{N}(t)=\mathcal{L}_{N} u^{N}(t) \\
u^{N}(0)=f
\end{array}\right.
$$

where $f \in \mathcal{D}^{1}$ with $\Lambda(f) \subset \Gamma_{N}$. According to Section 5.3 of [17], Eq. (4.4) has a mild solution $P_{t}^{N} f$ which satisfies

$$
P_{t}^{N} f\left(x^{N}\right)=S_{t}^{N} f\left(x^{N}\right)+\int_{0}^{t} S_{t-s}^{N}\left[\left\langle F^{N}, D P_{s}^{N} f\right\rangle_{H}\right]\left(x^{N}\right) d s,
$$


where $S_{t}^{N}$ is the Ornstein Uhlenbeck transition semigroup generated by the operator $\sum_{k \in \Gamma_{N}}\left[\beta_{k}^{\alpha} \partial_{k}^{\alpha}-\gamma_{k} x_{k} \partial_{k}\right]$. Moreover, we also have

$$
P_{t}^{N} f\left(x^{N}\right)=\mathbb{E}\left[f\left(X^{N}\left(t, x^{N}\right)\right)\right] .
$$

¿From the second step of the proof of [17, Theorem 5.7], we have

$$
\lim _{N \rightarrow \infty} P_{t}^{N} f\left(x^{N}\right)=P_{t} f(x),
$$

in particular, for any $f \in \mathcal{D}^{1}$ with $\Lambda(f) \subset \Gamma_{N}, t \geq 0, x \in H$, where $P_{t}$ is the transition semigroup defined in Theorem 2.4 .

4.2. Estimates and their proofs. The gradient estimates which are established here are of two different types. The first one is straightforward, and although formulated for (4.1), is true in a much more general situation. Moreover, it is stated in terms of the best Lipschitz constant of the nonlinearity $F$. The second one is true for the specific systems considered in the paper, requires more subtle considerations and is stated in terms of the supremum of $\{|F(x)| ; x \in H\}$.

Proposition 4.1. Let $P_{t}^{N}$ be the transition semigroup corresponding to the solution of the equation (4.1). Then

$$
\left\|D P_{t}^{N} f\right\|_{0} \leq e^{-\left(\gamma_{1}-L_{F}\right) t}\|D f\|_{0}, \quad f \in \mathcal{D}^{1} \text { with } \Lambda(f) \subset \Gamma_{N}, \quad t \geq 0 .
$$

Proof. Denoting by $X^{N}\left(t, x^{N}\right)$ the solution of Eq. (4.1) with initial data $x^{N}$, one has

$$
\left|X^{N}\left(t, x^{N}\right)-X^{N}\left(t, y^{N}\right)\right|_{H} \leq e^{-\left(\gamma_{1}-L_{F}\right) t}\left|x^{N}-y^{N}\right|_{H}, t \geq 0, x, y \in H,
$$

which implies $\lim _{\varepsilon \rightarrow 0} \sup \left|\frac{X^{N}\left(t, x^{N}+\varepsilon h^{N}\right)-X^{N}\left(t, x^{N}\right)}{\varepsilon}\right| \leq e^{-\left(\gamma_{1}-L_{F}\right) t}\left|h^{N}\right|, h \in H$. Hence, by the dominated convergence theorem one has

$$
\begin{aligned}
\left|D_{h} P_{t}^{N} f\left(x^{N}\right)\right| & \leq \mathbb{E}\left[\left|D f\left(X^{N}(t)\right)\right|_{H} \lim _{\varepsilon \rightarrow 0} \sup \left|\frac{X^{N}\left(t, x^{N}+\varepsilon h^{N}\right)-X^{N}\left(t, x^{N}\right)}{\varepsilon}\right|\right] \\
& \leq e^{-\left(\gamma_{1}-L_{F}\right) t}|| D f||_{0}|h|_{H} .
\end{aligned}
$$

Proposition 4.2. Let $P_{t}^{N}$ be the transition semigroup corresponding to the solution of the equation (4.1). There exists a positive constant $C$, depending on $\alpha,\left(\beta_{n}\right),\left(\gamma_{n}\right), \sigma$, such that

$$
\left\|D P_{t}^{N} f\right\|_{0} \leq C e^{-\omega t}\|D f\|_{0}, \quad f \in \mathcal{D}^{1}, \text { with } \Lambda(f) \subset \Gamma_{N}, \quad t \geq 0,
$$

where

$$
\omega=\frac{\gamma_{1}}{2}-\left(C_{0}\|F\|_{0} \Gamma(1-\sigma)\right)^{\frac{1}{1-\sigma}},
$$

and the constant $C_{0}$ was introduced in Theorem 2.6.

To prove Proposition 4.2, we need the following lemma. 
Lemma 4.3. For any $f \in \mathcal{D}^{1}$, with $\Lambda(f) \subset \Gamma_{N}$, one has:

(i) $\left|D S_{t}^{N} f\left(x^{N}\right)\right|_{H} \leq e^{-\gamma_{1} t}|| D f \|_{0}$,

(ii) $\left|D S_{t}^{N} f\left(x^{N}\right)\right|_{H} \leq \frac{C_{0} e^{-\gamma_{1} t / 2}}{t^{\sigma}}\|f\|_{0}$,

for all $x \in H, N \geq 1, t>0$ (where $C_{0}=\hat{c} \int_{\mathbb{R}} \frac{\left(p_{\alpha}^{\prime}(z)\right)^{2}}{p_{\alpha}(z)} d z$, see Theorem [2.6).

Proof. Note that, for any vector $h \in \sum_{k=1}^{N} h_{k} e_{k}, x \in H$, we have

$$
\left\langle D S_{t}^{N} f\left(x^{N}\right), h\right\rangle=\left\langle D S_{t} f\left(x^{N}\right), h\right\rangle,
$$

where $S_{t}$ is the Ornstein-Uhlenbeck semigroup acting on $C_{b}(H, \mathbb{R})$ associated to $X(t, x)$ when $F=0$. We know that

$$
S_{t} f(x)=\int_{H} f\left(e^{t A} x+y\right) \mu_{t}^{0}(d y),
$$

where $\mu_{t}^{0}$ is the law of the random variable $Z_{A}(t)=\int_{0}^{t} e^{(t-s) A} d Z_{s}$. By differentiating under the integral sign in (4.8) we immediately get the first assertion (remark that $\left\|e^{t A}\right\| \leq e^{-\gamma_{1} t}, t \geq 0$, where $\left\|e^{t A}\right\|$ denotes the operator norm of $\left.e^{t A}\right)$.

As for the second assertion, recall the gradient estimate from [17, Theorem 4.14]

$$
\left|D S_{t} f(x)\right|_{H} \leq 8 c_{\alpha}\left(\sup _{k \geq 1} \frac{e^{-\gamma_{k} t} \gamma_{k}^{1 / \alpha}}{\beta_{k}}\right)\|f\|_{0}, \quad f \in C_{b}(H, \mathbb{R}), \quad t>0,
$$

where

$$
c_{\alpha}=\frac{1}{8} \int_{\mathbb{R}} \frac{\left(p_{\alpha}^{\prime}(z)\right)^{2}}{p_{\alpha}(z)} d z
$$

According to (2.9) we have

$$
\left|D S_{t} f(x)\right|_{H} \leq 8 c_{\alpha} \frac{\hat{c} e^{-\gamma_{1} t / 2}}{t^{\sigma}}\|f\|_{0}, t>0
$$

and the assertion follows.

Proof of Proposition 4.2. By (4.5) and Lemma 4.3, we have, for any $N \in \mathbb{N}$,

$$
\left|D P_{t}^{N} f\left(x^{N}\right)\right| \leq e^{-\frac{\gamma_{1}}{2} t}|| D f\left\|_{0}+\int_{0}^{t} \frac{C_{0} e^{-\frac{\gamma_{1}}{2}(t-s)}}{(t-s)^{\sigma}}\right\|\left\langle F^{N}, D P_{s}^{N} f\right\rangle_{H} \|_{0} d s
$$

where $C_{0}$ is defined in Lemma 4.3, therefore,

$$
\left\|D P_{t}^{N} f\right\|_{0} \leq e^{-\frac{\gamma_{1}}{2} t}\|D f\|_{0}+\int_{0}^{t} \frac{C_{0} e^{-\frac{\gamma_{1}}{2}(t-s)}}{(t-s)^{\sigma}}\|F\|_{0}\left\|D P_{s}^{N} f\right\|_{0} d s
$$

Writing $v^{N}(t)=e^{\frac{\gamma_{1}}{2} t}\left\|D P_{t}^{N} f\right\|_{0}$, we have from the above inequality

$$
v^{N}(t) \leq\|D f\|_{0}+\int_{0}^{t} \frac{C_{0}\|F\|_{0}}{(t-s)^{\sigma}} v^{N}(s) d s
$$


Now we use the following Henry's estimate (see [11]): let $a \geq 0, b \geq 0$ and $\beta>0$ and consider a non-negative locally integrable function $u$ on $[0, T)$ such that

$$
u(t) \leq a+b \int_{0}^{t}(t-s)^{\beta-1} u(s) d s, \quad t \in[0, T),
$$

then we have $u(t) \leq a G_{\beta}(\theta t), t \in[0, T)$, where

$$
\theta=(b \Gamma(\beta))^{1 / \beta}, \quad G_{\beta}(z)=\sum_{n \geq 0} \frac{z^{n \beta}}{\Gamma(n \beta+1)}, \quad z \geq 0
$$

(note that $G_{\beta}(z) \sim \frac{1}{\beta} e^{z}$ as $\left.z \rightarrow+\infty\right)$.

In our case $u(t)=v^{N}(t), a=\|D f\|_{0}, b=C_{0}\|F\|_{0}$ and $\beta=1-\sigma$. Thus for a constant $C_{1}$, depending on $\sigma$, and all positive $t$ we get

Therefore

$$
v^{N}(t) \leq \frac{C_{1}\|D f\|_{0}}{1-\sigma} \exp \left\{\left(C_{0}\|F\|_{0} \Gamma(1-\sigma)\right)^{\frac{1}{1-\sigma}} t\right\}
$$

$$
\left\|D P_{t}^{N} f\right\|_{0} \leq \frac{C_{1}\|D f\|_{0}}{1-\sigma} \exp \left\{\left(C_{0}\|F\|_{0} \Gamma(1-\sigma)\right)^{\frac{1}{1-\sigma}} t-\frac{\gamma_{1}}{2} t\right\}
$$

and we get the assertion.

\section{Proof of Theorem 2.6}

From Theorem 2.5, the system (1.1) is ergodic and has a unique invariant measure $\mu$.

Note that it is enough to prove (2.10) for any $f \in \mathcal{D}^{1}$. Indeed then, approximating any function $f \in C_{b}^{1}(H)$ by a sequence $\left(f_{n}\right) \subset \mathcal{D}^{1}$ such that $f_{n} \rightarrow f$ and $D f_{n} \rightarrow D f$ pointwise with $\sup _{n>1}\left\|f_{n}\right\|_{C_{b}^{1}}<\infty$, we get easily the complete assertion. Let us fix $f \in \mathcal{D}^{1}$ and suppose that $\Lambda(f) \subset \Gamma_{N}$.

The crucial point of the proof is to apply the gradient estimate from Proposition 4.2, in the spirit of [15, Proposition 16.4].

Concerning the case (i), by using (4.7), we can show the exponential mixing by a similar argument as for the case (ii). So from now on we shall concentrate on the proof of the case (ii).

First let us suppose that $\alpha \in(1,2)$. Let

$$
Z_{A}^{N}(t)=\int_{0}^{t} e^{A(t-s)} d Z_{s}^{N}
$$

and write $Y^{N}(t)=X^{N}\left(t, x^{N}\right)-Z_{A}^{N}(t)$ so that

$$
Y^{N}(t)=e^{A t} x^{N}+\int_{0}^{t} e^{A(t-s)} F^{N}\left(X^{N}\left(s, x^{N}\right)\right) d s .
$$

For any $t_{2}>t_{1}>0$, writing $s=t_{2}-t_{1}$, by the gradient estimates of Proposition 4.2, we have, for any $x \in H$, 


$$
\begin{array}{rl}
\mid P_{t_{2}}^{N} f\left(x^{N}\right)-P_{t_{1}}^{N} & f\left(x^{N}\right)|=| \mathbb{E}\left[P_{t_{1}}^{N} f\left(X^{N}\left(s, x^{N}\right)\right)-P_{t_{1}}^{N} f\left(x^{N}\right)\right] \mid \\
& \leq\left\|D P_{t_{1}}^{N} f\right\|_{0} \mathbb{E}\left|X^{N}\left(s, x^{N}\right)-x^{N}\right|_{H} \\
& \leq C e^{-\omega t_{1}}|| D f \|_{0} \mathbb{E}\left|X^{N}\left(s, x^{N}\right)-x^{N}\right|_{H}
\end{array}
$$

where $C, \omega>0$ are given in Proposition 4.2. Now note that

$$
\left|X^{N}\left(t, x^{N}\right)-x^{N}\right|_{H} \leq\left|e^{A t} x^{N}-x^{N}\right|_{H}+\mid \int_{0}^{t} e^{A(t-s)} F^{N}\left(\left.X^{N}\left(s, x^{N}\right) d s\right|_{H}+\left|Z_{A}(t)\right|_{H} .\right.
$$

We have

$$
\left|\int_{0}^{t} e^{A(t-s)} F^{N}\left(X^{N}\left(s, x^{N}\right)\right) d s\right|_{H} \leq\|F\|_{0} \int_{0}^{\infty} e^{-\gamma_{1} s} d s \leq \frac{\|F\|_{0}}{\gamma_{1}},
$$

for any $\omega \in \Omega, x \in H$ and $t \geq 0$. Concerning $\left|Z_{A}(t)\right|_{H}$ remark that since $\alpha>1$ (see [17, Theorem 4.4])

$$
\mathbb{E}\left|Z_{A}(t)\right|_{H} \leq \tilde{c}_{1}\left(\sum_{n \geq 1}\left|\beta_{n}\right|^{\alpha} \frac{\left(1-e^{-\alpha \gamma_{n} t}\right)}{\alpha \gamma_{n}}\right)^{1 / \alpha} \leq \tilde{c}_{1}\left(\sum_{n \geq 1}\left|\beta_{n}\right|^{\alpha} \frac{1}{\alpha \gamma_{n}}\right)^{1 / \alpha}<+\infty, \quad t \geq 0 .
$$

It follows that

$$
\mathbb{E}\left|X^{N}\left(s, x^{N}\right)-x^{N}\right|_{H} \leq C_{2}\left(1+\left|x^{N}\right|_{H}\right),
$$

where $C_{2}$ does not depend on $N, s \geq 0$ and $x \in H$.

By (5.1) we get

$$
\left|P_{t_{2}}^{N} f\left(x^{N}\right)-P_{t_{1}}^{N} f\left(x^{N}\right)\right| \leq C_{3} e^{-\omega t_{1}}|| D f \|_{0}\left(1+\left|x^{N}\right|_{H}\right) .
$$

Passing to the limit as $N \rightarrow \infty$, we get (see the proof of Theorem 5.7 in [17])

$$
\left|P_{t_{2}} f(x)-P_{t_{1}} f(x)\right| \leq C_{3} e^{-\omega t_{1}}|| D f \|_{0}\left(1+|x|_{H}\right) .
$$

where $C_{3}$ does not depend on $t_{1}$. This estimate shows that $P_{t} f(x)$ converges to some constant exponentially fast as $t \rightarrow+\infty$. By ergodicity of the system, this constant must be $\mu(f)$.

Let us consider now $\alpha \in(0,1]$. For any $f \in C_{b}^{1}(H, \mathbb{R}), s \in(0,1]$, define $[f]_{s}$ by

$$
[f]_{s}:=\sup _{x \neq y} \frac{|f(x)-f(y)|}{|x-y|^{s}} .
$$

We have

$$
[f]_{s} \leq 2^{1-s} \sup _{x \neq y} \frac{|f(x)-f(y)|^{s}}{|x-y|^{s}}\|f\|_{0}^{1-s} \leq 2^{1-s}\|f\|_{0}^{1-s}\|D f\|_{0}^{s} .
$$


Now we choose $p \in(0, \alpha)$. Using that $\mathbb{E}\left|Z_{A}(t)\right|_{H}^{p}<\infty$ (see [17, Theorem 4.4]) and (5.2) with $s=p$ we get, arguing as before,

$$
\begin{array}{rl}
\mid P_{t_{2}}^{N} f\left(x^{N}\right)-P_{t_{1}}^{N} & f\left(x^{N}\right)|=| \mathbb{E}\left[P_{t_{1}}^{N} f\left(X^{N}\left(s, x^{N}\right)\right)-P_{t_{1}}^{N} f\left(x^{N}\right)\right] \mid \\
& \leq 2^{1-p}\left\|D P_{t_{1}}^{N} f\right\|_{0}^{p}\|f\|_{0}^{1-p} \mathbb{E}\left|X^{N}\left(s, x^{N}\right)-x\right|_{H}^{p} \\
& \leq 2^{1-p} C^{p} e^{-\omega p t_{1}}\|f\|_{C_{b}^{1}} \mathbb{E}\left|X^{N}\left(s, x^{N}\right)-x^{N}\right|_{H}^{p} .
\end{array}
$$

Since

$$
\mathbb{E}\left|Z_{A}(t)\right|_{H}^{p} \leq \tilde{c}_{p}\left(\sum_{n \geq 1}\left|\beta_{n}\right|^{\alpha} \frac{\left(1-e^{-\alpha \gamma_{n} t}\right)}{\alpha \gamma_{n}}\right)^{p / \alpha} \leq \tilde{c}_{p}\left(\sum_{n \geq 1}\left|\beta_{n}\right|^{\alpha} \frac{1}{\alpha \gamma_{n}}\right)^{p / \alpha}<+\infty, \quad t \geq 0,
$$

it follows that

$$
\mathbb{E}\left|X^{N}(s, x)-x^{N}\right|_{H}^{p} \leq C_{4}\left(1+\left|x^{N}\right|_{H}\right)^{p}
$$

where $C_{4}$ does not depend on $N, s \geq 0$ and $x \in H$. Finally we have

$$
\left|P_{t_{2}} f(x)-P_{t_{1}} f(x)\right| \leq C_{5} e^{-\omega p t_{1}}|| f \|_{C_{b}^{1}}\left(1+|x|_{H}\right)^{p},
$$

where $C_{5}$ does not depend on $t_{1}$. Arguing as before we complete the proof.

Acknowledgements. The authors thank the Newton Institute (Cambridge), where this paper was initiated, for its hospitality.

\section{REFERENCES}

1. S. Albeverio, V. Mandrekar, and B. Rüdiger, Existence of mild solutions for stochastic differential equations and semilinear equations with non-Gaussian Lévy noise, Stochastic Process. Appl. 119 (2009), no. 3, 835-863.

2. S. Albeverio, B. Rüdiger, and J. L. Wu, Invariant measures and symmetry property of Lévy type operators, Potential Anal. 13 (2000), no. 2, 147-168.

3. S. Albeverio, J. L. Wu, and T. S. Zhang, Parabolic SPDEs driven by Poisson white noise, Stochastic Process. Appl. 74 (1998), no. 1, 21-36.

4. Z. Brzezniak, B. Goldys, P. Imkeller, S. Peszat, E. Priola and J. Zabczyk, Time irregularity of generalized Ornstein-Uhlenbec processes, C. R. Acad. Sci. Paris Ser. Math. 348 (2010), 273-276.

5. P. Cannarsa and G. Da Prato, A semigroup approach to Kolmogoroff equations in Hilbert spaces,Appl. Math. Lett. 4 (1991), no. 1, 4952,

6. A. Chojnowska-Michalik, On processes of Ornstein-Uhlenbeck in Hilbert spaces, Stochastics 21 (1987), 251-286.

7. G. Da Prato and J. Zabczyk, Stochastic equations in infinite dimensions, Encyclopedia of Mathematics and its Applications, vol. 44, Cambridge University Press, Cambridge, 1992.

8. G. Da Prato and J. Zabczyk, Ergodicity for infinite-dimensional systems, London Mathematical Society Lecture Note Series, vol. 229, Cambridge University Press, Cambridge, 1996.

9. G. Da Prato and J. Zabczyk, Regular densities of invariant measures for nonlinear stochastic equations, J. Functional Analysis, 130 (1995), 427-449.

10. T. Funaki and B. Xie, A stochastic heat equation with the distributions of Lévy processes as its invariant measures, Stochastic Process. Appl. 119 (2009), no. 2, 307-326. 
11. D. Henry, Geometric theory of semilinear parabolic equations, Lecture Notes in Mathematics, 840. Springer-Verlag, Berlin-New York, 1981.

12. C. Marinelli and M. Röckner, Well-posedness and asymptotic behavior for stochastic reactiondiffusion equations with multiplicative poisson noise, arXiv:0903.3299 2 .

13. B. Øksendal, Stochastic partial differential equations driven by multi-parameter white noise of Lévy processes, Quart. Appl. Math. 66 (2008), no. 3, 521-537.

14. K.R. Parthasarathy, Probability measures on metric spaces, Academic Press. 1967.

15. S. Peszat and J. Zabczyk, Stochastic partial differential equations with Lévy noise, Encyclopedia of Mathematics and its Applications, vol. 113, Cambridge University Press, Cambridge, 2007, An evolution equation approach.

16. S. Peszat and J. Zabczyk, Stochastic heat and wave equations driven by an impulsive noise, Stochastic partial differential equations and applications VII, Lect. Notes Pure Appl. Math., vol. 245, Chapman \& Hall/CRC, Boca Raton, FL, 2006, pp. 229-242.

17. E. Priola and J. Zabczyk, Structural properties of semilinear spdes driven by cylindrical stable processes, published on line on Probab. Theory Related Fields (arXiv:0810.5063v1).

18. E. Priola and J. Zabczyk, On linear evolution equations with cylindrical Levy noise to appear in Proceedings "SPDE's and Applications - VIII", Quaderni di Matematica, Seconda Università di Napoli (arXiv:0908.0356v1).

19. A. Rusinek, Mean reversion for HJMM forward rate models, Adv. in Appl. Probab. 42 (2010), no. 2, 371-391.

20. L. Xu and B. Zegarliński, Ergodicity of the finite and infinite dimensional $\alpha$-stable systems, Stoch. Anal. Appl. 27 (2009), no. 4, 797-824.

21. L. Xu and B. Zegarlinski, Existence and exponential mixing of infinite white $\alpha$-stable systems with unbounded interactions, 2009, arXiv:0911.2866

Dipartimento di Matematica, Università di Torino, via Carlo Alberto 10, 10123 TORINO, ITALY

E-mail address: enrico.priola@unito.it

TU Berlin, Fakultät II, Institut für Mathematik, Str $\alpha \beta$ E Des $17 . \quad$ Juni 136 , D-10623 Berlin, Germany

E-mail address: xu@math.tu-berlin.de

Institute of Mathematics, Polish Academy of Sciences, P-00-950 Warszawa, POLAND

E-mail address: zabczyk@impan.pl 